\title{
Correction to: Production diversification, dietary diversity and consumption seasonality: panel data evidence from Nigeria
}

Habtamu Yesigat Ayenew ${ }^{1 *}$, Sibhatu Biadgilign², Lena Schickramm ${ }^{1}$, Getachew Abate-Kassa ${ }^{1}$ and Johannes Sauer ${ }^{1}$

\section{Correction}

It has been highlighted that the original article [1] contained a typesetting mistake in the name of Sibhatu Biadgilign. This was incorrectly captured as Biadigilign in the original publication which has since been updated.

\footnotetext{
Author details

${ }^{1}$ Agricultural Production and Resource Economics, Technical University Munich, Freising, Germany. ${ }^{2}$ Public Health Consultant Ethiopia, Addis Ababa, Ethiopia.
}

Received: 23 August 2018 Accepted: 23 August 2018

Published online: 29 August 2018

\section{Reference}

1. Ayenew HY, et al. Production diversification, dietary diversity and

consumption seasonality: panel data evidence from Nigeria. BMC Public Health. 2018;18:988. https://doi.org/10.1186/s12889-018-5887-6.

\footnotetext{
* Correspondence: habtamu.ayenew@tum.de

${ }^{1}$ Agricultural Production and Resource Economics, Technical University

Munich, Freising, Germany
} 